\title{
Multiobjective Optimization by Decision Diagrams
}

\author{
David Bergman and Andre A. Cire \\ Version Post-print/accepted manuscript \\ Citation Bergman D., Cire A.A. (2016) Multiobjective Optimization by Decision \\ (published version) Diagrams. In: Rueher M. (eds) Principles and Practice of Constraint \\ Programming. CP 2016. Lecture Notes in Computer Science, vol 9892. \\ Springer, Cham
}

Publisher's Statement The final publication is available at link.springer.com via http://dx.doi.org/ 10.1007/978-3-319-44953-1_6.

How to cite TSpace items

\begin{abstract}
Always cite the published version, so the author(s) will receive recognition through services that track citation counts, e.g. Scopus. If you need to cite the page number of the author manuscript from TSpace because you cannot access the published version, then cite the TSpace version in addition to the published version using the permanent URI (handle) found on the record page.
\end{abstract}

This article was made openly accessible by $U$ of $T$ Faculty. Please tell us how this access benefits you. Your story matters. 


\title{
Multiobjective Optimization by Decision Diagrams
}

\author{
David Bergman ${ }^{1}$ and Andre A. Cire ${ }^{2}$ \\ 1 Department of Operations and Information Management, University of Connecticut \\ david.bergman@business . uconn.edu \\ 2 Department of Management, University of Toronto Scarborough \\ acire@utsc.utoronto.ca
}

\begin{abstract}
In this paper we present a technique for solving multiobjective discrete optimization problems using decision diagrams. The proposed methodology is related to an algorithm designed for multiobjective optimization for dynamic programming, except utilizing decision diagram theory to reduce the state space, which can lead to orders of magnitude performance gains over existing algorithms. The decision diagrambased technique is applied to knapsack, set covering, and set partitioning problems, exhibiting improvements over state-of-the-art general-purpose multiobjective optimization algorithms.
\end{abstract}

Keywords: Decision diagrams; Multiobjective optimization; Multicriteria decision making; Multicriteria shortest path

Keywords: Discrete Multiobjective Optimization, Decision Diagrams. Pre-Print - Principles and Practice of Constraint Programming, 2016. The final publication is available at Springer via https://doi.org/10.1007/978-3-319-44953-1_6

\section{Introduction}

Automated decision making, by its very nature, requires the consideration of a multitude of objectives. Multiobjective optimization, also known as multiobjective programming, vector optimization, multi-criteria optimization, multiattribute optimization or Pareto optimization, has a rich history, dating back to the emergence of rigorous mathematical programming [21]. Several books have been written on the topic $[11,28,14]$, and many in-depth surveys $[34,37,12,13,15]$.

The present paper concerns multiobjective discrete optimization problems (MODO), where the variables of the problem are discrete and the number of objectives is $p \geq 2$. An efficient solution to a MODO is one in which there is no other solution that improves, simultaneously, on each of the objectives. The vector corresponding to the objectives of an efficient solution is called a nondominated solution. The set of efficient solutions is known as the efficient set and the set of all nondominated solution is known as the nondominated set. The goal of a MODO, for the purposes of this paper, is to enumerate the nondominated set. 
Perhaps the most commonly studied technique for identifying all nondominated solutions for MODOs is the $\epsilon$-constraint method [18], having well-known properties for more than two objectives [11]. The $\epsilon$-constraint method was first used by Laumanns et al. [24], where an adaptive search over weighted objectives was proposed. Özlen et al. [27,29] and Kirlik and Sayın [23] provide algorithmic improvements and are the basis for the comparison in the computational results presented in this paper. Other utilized scalarization techniques include Benson's method [3] (a combination of the weight-sum technique and the $\epsilon$ constraint method) and the augmented weighted Chebychev method [32]. The main drawback of these techniques is that a discrete optimization problem, often NP-hard in practice, must be solved several times so as to enumerate the necessary scalarizations, leading to the bottleneck of their procedures.

In this paper, we propose an alternative method that creates a single binary decision diagram (BDD) $[25,1,9,10]$ which represents all feasible solutions to the problem as a directed acyclic graph, and then employs multicriteria shortest path problem (MSP) algorithms to enumerate the nondominated set. This technique therefore transforms the problem into that of (1) finding the exact BDD for the constraint set of the MODO, and (2) using MSP algorithms to find the set of nondominated solutions.

The utilization of decision diagrams in optimization is recent and focusses on the use of BDDs for a variety of purposes [5]. In this research stream, top-down compilation methods for set covering problems $[7,6]$ and set packing problems $[6,4]$ have been investigated, along with methods for creating BDDs for knapsack problems [2]. On the other hand, published articles on MSP are abundant. Research on MSP started in the 1970s [35,19] and has typically concentrated on two objectives (see Raith and Ehrgott [30] for details). A survey on exact methods for the MSP is provided by Garroppo et al. [17].

Our methodology is primarily based on the work by Loui [26], who proposes a multidimensional labeling technique for dynamic programming (DP) models with stochastic or multidimensional weights. Such techniques have been considered in several papers [30] and are known in the DP community (see, e.g., Bertsekas [8], §2.3.4), often being applied to multiobjective knapsack algorithms, as by Figueira et al. [31]. Nonetheless, labeling algorithms are typically prohibitive in practice due to the curse of dimensionality of DP models, as the state space often grows too quickly in practice.

BDDs are closely related to dynamic programming and, as in DPs, they also reduce discrete optimization to shortest path problems. However, it has been shown that the state-space associated with a BDD can be much more compact than that of a similar DP model [20], which is key to our methodology. Our major contribution, hence, is the introduction of a MSP labeling algorithms for decision diagrams. This yields an alternative, simple method for enumerating the nondominated set of a multiobjective problem. The technique is applicable to a number of discrete optimization problems, and our numerical study indicates that it can be orders of magnitude faster than state-of-the-art techniques. 


\section{Multiobjective Discrete Optimization}

A MODO $\mathcal{M}$ is specified by a set of $p$ objective functions $f^{j}: \mathbb{R}^{n} \rightarrow \mathbb{R}$, for $j=$ $1, \ldots, p$, and a feasible set $\mathcal{X}$. In this paper it is assumed that each objective function is additively separable or, more simply, linear, so that $f^{j}(x)=\sum_{i=1}^{n} c_{i}^{j} x_{i}$. The feasible set $\mathcal{X}$ is discrete - this paper will focus on binary optimization problems so that $\mathcal{X} \subseteq \mathbb{B}^{n}$, although the techniques are easily generalizable. Each $x \in \mathcal{X}$ is said to be a feasible solution.

Let $f$ be the set of objective functions $f: \mathbb{R}^{n} \rightarrow \mathbb{R}^{p}$ (assumed to be maximized unless otherwise specified). Each solution $x \in \mathcal{X}$ is mapped through the function $f$ into a corresponding objective vector $y=f(x) \in \mathbb{R}^{p}$. The set of objective vectors $\mathcal{Y}=\{f(x): x \in \mathcal{X}\}$ resulting from feasible solutions is the objective space. A solution $x^{*}$ is called an efficient solution if there exists no other feasible solution $x^{\prime}$ such that, for all $j, f^{j}\left(x^{\prime}\right) \geq f^{j}\left(x^{*}\right)$ with $f^{j^{\prime}}\left(x^{\prime}\right)>f^{j^{\prime}}\left(x^{*}\right)$ for some $j^{\prime}$. For an efficient solution $x^{*}$ the vector $f\left(x^{*}\right)$ is referred to as a nondominated solution. The efficient set, denoted by $\mathcal{X}_{\mathrm{E}}$, is the set of all efficient solutions and its image, denoted by $\mathcal{Y}_{\mathrm{N}}=\left\{y: y=f(x)\right.$ for some $\left.x \in \mathcal{X}_{\mathrm{E}}\right\}$, is the nondominated set. The (typical) goal of MODO and the focus of this paper is to obtain $\mathcal{Y}_{\mathrm{N}}$.

\section{Binary Decision Diagrams}

A binary decision diagrams $\mathrm{BDD} B=(n, U, A, \ell, d)$ is a layered-acyclic digraph composed of node set $U$ and $\operatorname{arcs} A$. The mapping $\ell: U \rightarrow\{1,2, \ldots, n+1\}$ partitions the nodes into $n+1$ layers $L_{i}:=\{u \in U: \ell(u)=i\}, i=1,2, \ldots, n+1$. Layers $L_{1}$ and $L_{n+1}$ have cardinality one, with the single nodes in these layers denoted by root $\mathbf{r}$ and terminal $\mathbf{t}$, respectively. Each arc $a \in A$ leaves a tail node $t(a)$ and enters a head node $h(a)$, where $t(a), h(a) \in U$. It is assumed that $\ell(h(a))=\ell(t(a))+1$, so that each arc connects nodes in adjacent layers. Also, each node $u$ has at most one out-directed arc $a$ with arc-domain $d \in\{0,1\}$. An arc leaving node $u$ is denoted by $a_{0}(u)$ if $d=0$ and by $a_{1}(u)$ otherwise. The width of a layer is $w\left(L_{i}\right):=\left|L_{i}\right|$, and the width of $B$ is $w(B):=\max _{i \in\{1, \ldots, n+1\}} w\left(L_{i}\right)$. Finally, the size of a BDD is $|U|$.

A BDD represents a set of binary vectors in the following way. Each arcspecified path $p=\left(a_{1}, a_{2}, \ldots, a_{k}\right)$ represents the vector $x(p)=\left(d\left(a_{1}\right), d\left(a_{2}\right), \ldots\right.$, $\left.d\left(a_{k}\right)\right)$. Any path from $\mathbf{r}$ to $\mathbf{t}$ thereby corresponds to a vector in $\mathbb{B}^{n}$. Let $\mathcal{P}(B)$ be the set of arc-specified paths from $\mathbf{r}$ to $\mathbf{t}$. Define $\operatorname{Sol}(B)$ as the set of binary vectors (called solutions) corresponding to $\operatorname{arc}$-specified $\mathbf{r}-\mathbf{t}$ paths:

$$
\operatorname{Sol}(B)=\left\{x(p) \in \mathbb{B}^{n}: p \in \mathcal{P}(B)\right\} .
$$

BDDs can be used to represent the feasible set of a MODO $\mathcal{M}$ through relating $\operatorname{Sol}(B)$ with the set of feasible solutions $\mathcal{X}$ of $\mathcal{M}$. BDD $B$ is said to be an exact $B D D$ for $\mathcal{M}$ if $\operatorname{Sol}(B)=\mathcal{X}$.

Fix $u, v \in U$ for which $\ell(u)<\ell(v)$. Let $B_{u, v}$ be the BDD obtained by removing from $B$ any nodes and arcs that do not lie on any directed path from 
$u$ to $v$. A BDD is said to be reduced if for all $i=1, \ldots, n$ and any two nodes $u, u^{\prime} \in L_{i}$, the BDDs $B_{u, \mathbf{t}}$ and $B_{u^{\prime}, \mathbf{t}}$ are such that $\mathbf{S o l}\left(B_{u, \mathbf{t}}\right) \neq \mathbf{S o l}\left(B_{u^{\prime}, \mathbf{t}}\right)$.

It is well-known that for any set of solutions $\mathcal{X}$, with a specified ordering of the variables given, there is a unique reduced BDD [9]. The reduction of a BDD can be performed efficiently by a simple bottom-up $O(|U| \log (|U|))$ algorithm [36]. It often has dramatic effects on the size of the BDD and the calculation of the nondominated set.

Suppose $\tilde{\mathcal{X}} \subseteq \mathbb{B}^{4}$ is given by the following set of solutions

$$
\begin{array}{r}
\tilde{\mathcal{X}}=\{(0,0,0,0)(0,0,0,1)(0,0,1,0)(0,1,0,0)(0,1,0,1)(0,1,1,0) \\
(1,0,0,0)(1,0,0,1)(1,0,1,0)(1,1,0,0)(1,1,1,0)\}
\end{array}
$$

Consider the BDD $\tilde{B}$, depicted in Figure 1. Dashed/solid arcs correspond to arcs with arc-domain $0 / 1 . \tilde{B}$ is an exact BDD for $\tilde{\mathcal{X}}$ - each path from $\mathbf{r}$ to $\mathbf{t}$ corresponds to a solution in $\tilde{\mathcal{X}}$ and vice versa so that $\operatorname{Sol}(\tilde{B})=\tilde{\mathcal{X}}$.

We refer the reader to the work by Bergman et al. [5] for BDD compilation procedures for general optimization problems.
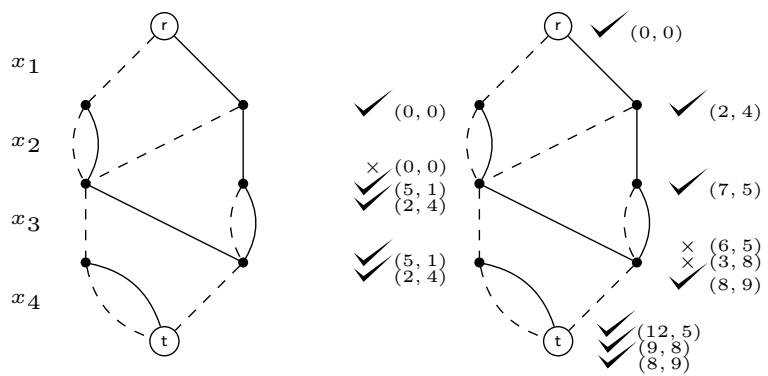

Fig. 1. Exact BDD for $\tilde{\mathcal{X}}$

\section{Determining the Nondominated Set}

In this section we propose a technique which generalizes previous works on singleobjective optimization problems using BDDs [5]. Namely, suppose a MODO $\mathcal{M}$ has one objective function (i.e., $p=1$ ) with $f^{1}(x)=\sum_{i=1}^{n} c_{i}^{1} x_{i}$, and assume $\mathcal{X}$ is represented by a BDD $B$. To optimize $f^{1}$, we associate an arc-value $v(a)$ with each arc $a \in L_{i}, i=1, \ldots, n$, where $v(a)=c_{i}^{1}$ if $d(a)=1$, and $v(a)=0$ otherwise. Any longest $\mathbf{r}$ to $\mathbf{t}$ path $p$ with respect to $v$ yields an optimal solution $x(p)$, with the corresponding optimal value equal to the length of the path.

Let $\mathcal{M}$ be a MODO with $p \geq 2$. Given an exact BDD $B$ for $\mathcal{X}$, we apply Algorithm 1 to determine $\mathcal{Y}_{\mathrm{N}}$. The algorithm traverses $B$ in a top-down fashion and associates a state $s$ with each node $u$, representing the set of nondominated solutions in $\mathbb{R}^{\ell(u)-1}$ contained in $B_{\mathbf{r}, u}$; at the end of the execution, $s(\mathbf{t})=\mathcal{Y}_{\mathrm{N}}$. 


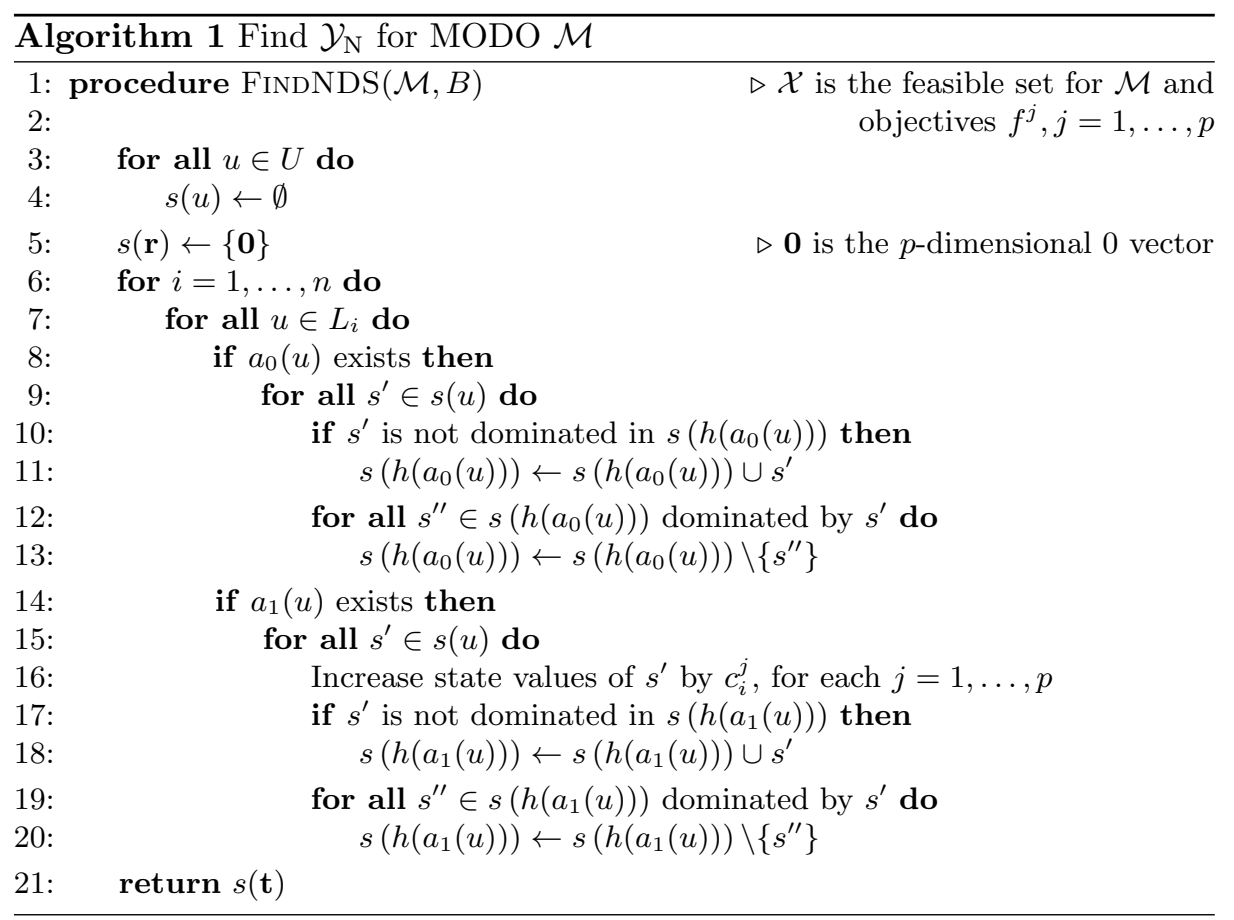

The algorithms initializes $s(u)$ for each node $u \in U \backslash\{\mathbf{r}\}$ to $\emptyset$, and initializes $s(\mathbf{r})$ to $\{\mathbf{0}\}$ (the $p$-dimensional vector with 0 s in each coordinate). Having set the states of each node in $L_{i}$ the algorithm proceeds to determine the states for the nodes in $L_{i+1}$. For each node in $L_{i}$ and each arc directed out of $u$, the algorithm checks whether or not a nondominated solution will arise from extending solutions ending at $u$ with the arc domain of the arc.

For each layer $L_{i}$, if the arc under consideration is a 0 -arc, then any nondominated solution will not be affected by setting $x_{i}=0$. Therefore, each nondominated solution in $s(u)$ is considered as a possible nondominated solution in $s(h(a))$. If it is nondominated, the solution is added to $s(h(a))$, and otherwise omitted. The process is analogous for the case of a 1-arc, except that any potential nondominated solution will have its objective value increased by $c_{i}^{j}$ for each $j=1, \ldots, p$. Finally, if a solution $s^{\prime}$ is added to the state of a node, we must verify if any solutions $s^{\prime \prime}$ in that same state are now dominated by $s^{\prime}$, removing them if that is the case.

Suppose, for example, that a MODO $\mathcal{M}^{\prime}$ is specified with $\tilde{\mathcal{X}}$ defined as above and 2 objective functions, $f^{1}(x)=2 x_{1}+5 x_{2}+x_{3}+7 x_{4}, f^{2}(x)=4 x_{1}+x_{2}+4 x_{3}+$ $4 x_{4}$. Figure 1 shows the result of applying Algorithm 1 to the BDD in Figure 1. Each node $u$ is labeled with a set of vectors in the objective space. Those marked with a $\checkmark$ compose $s(u)$ and those marked with a $\times$ are those candidates for $s(u)$ that are determined to be dominated by some solution in $s(u)$. As a concrete example, consider the node $u$ marked with the three vectors $(0,0),(5,1)$ and 
$(2,4) .(0,0)$ is first added to $s(u)$ because of the zero-arc directed at $u$ from the node immediately above it. Then, when the one-arc from that same node is considered, it generates the vector $(0,0)+(5,1)=(5,1)$, eliminating $(0,0)$ because $(5,1)$ dominates it. Then, the zero-arc from the right-most node on the previous layer is examined, generating vector $(2,4)+(0,0)=(2,4)$. The set $\{(5,1),(2,4)\}$ is a collection of vectors, none of which dominate any other, and so $(2,4)$ is added to $s(u)$. At the conclusion of the algorithm, $\mathbf{t}$ is labeled with $s(\mathbf{t})=\{(12,5),(9,8),(8,9)\}$, the set $\mathcal{Y}_{\mathrm{N}}$ for $\mathcal{M}^{\prime}$. The proof of correctness of Algorithm 1 follows immediately, e.g., from the results of Figueira et al. [31] and Loui [26].

\section{Numerical Study}

In this section we evaluate the empirical performance of the proposed multiobjective methodology on three classical optimization problems: knapsack, set covering, and set packing. Our key performance metric is the time to enumerate the complete set of nondominated solutions. We compare our approach with another general-purpose MODO solver developed by Kirlik and Sayın [23], an $\epsilon$-constraint scalarization method which is currently regarded as one of the state of the art approaches for MODOs. We note in passing that we have also investigated other MODO solvers, such as the one proposed by Özlen et al. [29], but the method by Kirlik and Saym was the best performing and numerically stable method across all solvers tested in our empirical setting.

The experiments ran on an $\operatorname{Intel}(\mathrm{R}) \mathrm{Xeon}(\mathrm{R}) \mathrm{CPU}$ E5-2640 v3 at $2.60 \mathrm{GHz}$ with 128 GB RAM. The BDD method was implemented in $\mathrm{C}++$ and compiled with GCC 4.8.4. Our source code and all tested instances are available at http://www.andrew.cmu.edu/user/vanhoeve/mdd/. The source code for the technique by Kirlik and Saym was downloaded from http://home.ku.edu.tr/ moolibrary/ and linked with ILOG CPLEX 12.6.3 [22]. A time limit of 3,600 seconds was allotted in all cases.

Multicriteria Knapsack. Given $n$ items, a capacity $W>0$, and for each item $i$ a weight $w_{i}>0$ and $p$ profits $v_{i}^{1}, v_{i}^{2}, \ldots, v_{i}^{p}>0$, the multicriteria knapsack problem (MKP) is: $\max \left\{\sum_{i=1}^{n} v_{i}^{j} x_{i}, j=1, \ldots, p: \sum_{i=1}^{n} w_{i} x_{i} \leq W, x \in\{0,1\}^{n}\right\}$.

We generated random MKP instances following the procedure by Kirlik and Sayın [23]. The values $v_{i}^{j}$ and $w_{i}$ were drawn uniformly at random from the set $\{1, \ldots, 1000\}$, and $W=\left\lceil 0.5 \sum_{i=1}^{n} w_{i}\right\rceil$. Due to the growth of the nondominated set, for $p=3$, we considered $n=\{10, \ldots, 100\}$; for $p=4, n \in\{10, \ldots, 70\}$; for $p=5,6$, and $7, n \in\{20,30,40\}$. We generated 10 instances per pair $(n, p)$.

Table 1 presents the average cardinalities of the nondominated sets $\left(\left|\mathcal{Y}_{\mathrm{N}}\right|\right)$ and average solution times (within solved instances) for $p=3,4,5$, where Kirlik and BDD denotes the method by Kirlik and Sayı [23] and the BDD technique, respectively. Figure 2(a) depicts a scatter plot comparing solution times for all instances, where the size of a point is proportional to $n$. The BDD method is substantially faster and more robust than Kirlik, in particular when $p$ is large. 
Table 1. Average solution times (in seconds) for knapsack problems for $p=3,4,5$. Number in parentheses indicate instances unsolved within the time limit (out of 10).

\begin{tabular}{|c|c|c|c|c|c|c|c|c|c|}
\hline \multirow[b]{2}{*}{$n$} & \multicolumn{3}{|l|}{$p=3$} & \multicolumn{3}{|l|}{$p=4$} & \multicolumn{3}{|l|}{$p=5$} \\
\hline & $\left|\mathcal{Y}_{N}\right|$ & Kirlik & $\mathrm{BDD}$ & $\left|\mathcal{Y}_{N}\right|$ & Kirlik & BDD & $\left|\mathcal{Y}_{N}\right|$ & Kirlik & $\mathrm{BDD}$ \\
\hline 10 & 9 & 0.10 & 0.01 & 14 & 0.28 & 0.01 & 22 & 0.04 & 0.01 \\
\hline 20 & 37 & 0.85 & 0.03 & 79 & 7.22 & 0.04 & 241 & $1,319.48^{(7)}$ & 0.04 \\
\hline 30 & 113 & 4.95 & 0.17 & 397 & $466.40^{(1)}$ & 0.26 & 972 & (10) & 0.47 \\
\hline 40 & 370 & 24.71 & 0.95 & 1,278 & $217.38^{(9)}$ & 2.39 & 4,943 & (10) & 18.06 \\
\hline 50 & 598 & 50.47 & 3.64 & 3,374 & $(10)$ & 27.87 & & & \\
\hline 60 & 1,080 & 120.21 & 12.52 & 6,624 & (10) & 166.34 & & & \\
\hline 70 & 1,325 & 154.50 & 32.69 & 14,696 & (10) & 1,164 & & & \\
\hline 80 & 2,575 & 454.46 & 120 & & & & & & \\
\hline 90 & 3,847 & 912.47 & 350 & & & & & & \\
\hline 100 & 4,248 & $1,070^{(1)}$ & $551^{(1)}$ & & & & & & \\
\hline
\end{tabular}

In all cases, the time to create the BDD is only $5 \%$ of the total BDD time, and therefore the bottleneck is the computation of Algorithm 1. Finally, Figure 2(b) depicts a scatter plot comparing solution times of reduced and nonreduced BDDs for $n \in\{40,50,60\}$ and $p=4$, emphasizing the importance of reducing the number of nodes of the BDD before performing Algorithm 1 (which, on average, take less than a second on all tested cases). This saves approximately a half of an order of magnitude on the total computation time.

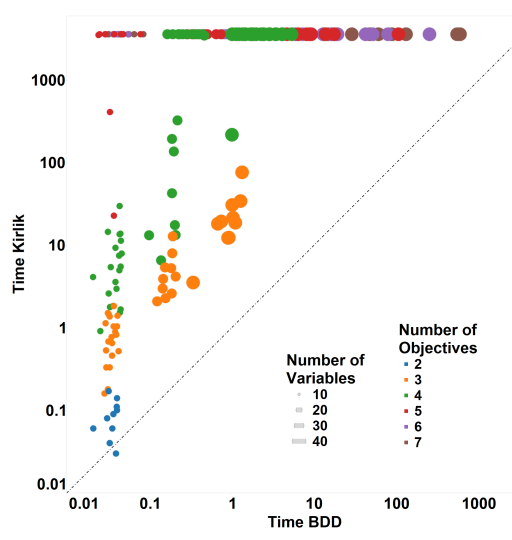

(a) Solution times.

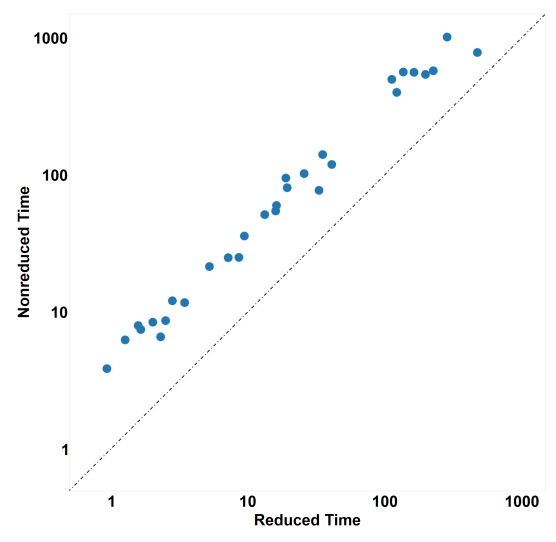

(b) Reduced and non-reduced.

Fig. 2. (a) Solution time comparison between Kirlik and BDD (in logarithmic scale), and (b) Solution times for reduced and non-reduced BDDs for the MKP. 
Multicriteria Set Covering and Set Packing. Let $A$ be a $0-1 m \times n$ constraint matrix, and let $c^{1}, \ldots, c^{p}$ be the $p$ cost vectors in $\mathbb{R}^{n}$. The multicriteria set covering problem (MSCP) is defined as $\min \left\{\left(c^{j}\right)^{T} x, j=1, \ldots, p: A x \geq 1, x \in\{0,1\}^{n}\right\}$. The multicriteria set packing problem (MSPP) is similar to the MSCP and is written as $\max \left\{\left(c^{j}\right)^{T} x, j=1, \ldots, p: A x \leq 1, x \in\{0,1\}^{n}\right\}$. The multiobjective variants pose a considerably more difficult challenge [16].

We performed experiments on random instances generated as in Stidsen et al. [33]. Specifically, we considered $n \in\{100,150\}, m=n / 5$, and in our case we fixed 10 variables per constraint, i.e., for every $k=1, \ldots, m, 10$ elements of the $k$-th row of $A$ were chosen to be equal to one uniformly at random. 10 instances were created per pair $(n, p)$. The objective coefficients were generated as they were for the MKP. The reduced BDDs for the MSPP and MSCP were compiled according to Bergman et al. [7,5].

Table 2 presents the average cardinalities of the nondominated sets and the average solution times. As before, BDD is substantially faster and more robust than Kirlik, especially as the number of objective function increases. The BDDs performed particularly well for set packing problems, since they are typically much more compact when compared to the BDD representing MSCPs instances. The time to compile the BDDs was also less than $5 \%$ of the total BDD time, as in the MKP case.

Table 2. Average solution times (in seconds) for MSCPs and MSPPs problems. Number in parentheses indicate instances unsolved within the time limit (out of 10).

\begin{tabular}{|c|c|c|c|c|c|c|c|}
\hline \multirow[b]{2}{*}{$n$} & \multirow[b]{2}{*}{$p$} & \multicolumn{3}{|c|}{ Set Covering (MSCP) } & \multicolumn{3}{|c|}{ Set Packing (MSPP) } \\
\hline & & $\left|\mathcal{Y}_{\mathrm{N}}\right|$ & Kirlik & $\mathrm{BDD}$ & $\left|\mathcal{Y}_{\mathrm{N}}\right|$ & Kirlik & BDD \\
\hline \multirow[t]{3}{*}{100} & 3 & 117 & 5.91 & 5.46 & 164 & 5.61 & 5.07 \\
\hline & 4 & 428 & 282.19 & 7.03 & 551.10 & 321.64 & 5.12 \\
\hline & 5 & 1,171 & $82.79^{(9)}$ & 7.87 & $1,211.90$ & $(10)$ & 5.35 \\
\hline \multirow[t]{3}{*}{150} & 3 & 305 & 23.99 & 110.50 & 336.00 & 12.81 & 8.37 \\
\hline & 4 & 1,178 & $1,228.18^{(2)}$ & $248.44^{(2)}$ & 4,557 & $3,025.52^{(8)}$ & 22.24 \\
\hline & 5 & 4,711 & (10) & $329.49^{(5)}$ & 9,213 & (10) & 49.18 \\
\hline
\end{tabular}

\section{Conclusions}

This paper proposes an algorithm for solving general multiobjective discrete optimization problems using decision diagrams. We utilize decision diagrams to represent, exactly, the feasible set of the problem, and then uses a multicriteria shortest path algorithm for finding the set of nondominated solutions. The algorithm is applied to three classical discrete optimization problems, and com- 
putational methods indicate that the proposed method is superior to a state-ofthe-art multiobjective technique, often providing orders of magnitude speedups.

Acknowledgements: This research was supported in part by the Natural Sciences and Engineering Research Council of Canada (NSERC), Discovery Grant.

\section{References}

1. S. B. Akers. Binary decision diagrams. IEEE Transactions on Computers, C27:509-516, 1978.

2. Markus Behle. On threshold bdds and the optimal variable ordering problem. Journal of Combinatorial Optimization, 16(2):107-118, 2007.

3. H.P. Benson. Existence of efficient solutions for vector maximization problems. Journal of Optimization Theory and Applications, 26(4):569-580, 1978.

4. D. Bergman, A. A. Ciré, W.-J. van Hoeve, and J. N. Hooker. Variable ordering for the application of BDDs to the maximum independent set problem. In Nicolas Beldiceanu, Narendra Jussien, and Eric Pinson, editors, CPAIOR, volume 7298 of Lecture Notes in Computer Science, pages 34-49. Springer, 2012.

5. D. Bergman, A. A. Cire, W.-J. van Hoeve, and J. N. Hooker. Discrete optimization with decision diagrams. INFORMS Journal on Computing, 28(1):47-66, 2016.

6. D. Bergman, A. A. Cire, W.-J. Jan van Hoeve, and T. H. Yunes. BDD-based heuristics for binary optimization. Journal of Heuristics, 20(2):211-234, 2014.

7. D. Bergman, W.-J. van Hoeve, and J.N. Hooker. Manipulating MDD Relaxations for Combinatorial Optimization. In Tobias Achterberg and J. Beck, editors, Integration of AI and OR Techniques in Constraint Programming for Combinatorial Optimization Problems, volume 6697 of Lecture Notes in Computer Science, chapter 5, pages 20-35. Springer Berlin / Heidelberg, Berlin, Heidelberg, 2011.

8. Dimitri P. Bertsekas. Dynamic Programming and Optimal Control. Athena Scientific, 2nd edition, 2000.

9. R. E. Bryant. Graph-based algorithms for boolean function manipulation. IEEE Transactions on Computers, C-35:677-691, 1986.

10. R. E. Bryant. Symbolic boolean manipulation with ordered binary decision diagrams. ACM Computing Surveys, 24:293-318, 1992.

11. V. Chankong and Y. Y. Haines. Multiobjective Decision Making: Theory and Methodology. Elsevier Science, 1983.

12. A. Chinchuluun and P. M. Pardalos. A survey of recent developments in multiobjective optimization. Annals of Operations Research, 154(1):29-50, 2007.

13. C. A. Coello. An updated survey of ga-based multiobjective optimization techniques. ACM Comput. Surv., 32(2):109-143, June 2000.

14. K. Deb. Multi-Objective Optimization Using Evolutionary Algorithms. John Wiley \& Sons, Inc., New York, NY, USA, 2001.

15. M. Ehrgott. A discussion of scalarization techniques for multiple objective integer programming. Annals of Operations Research, 147(1):343-360, 2006.

16. Kostas Florios and George Mavrotas. Generation of the exact pareto set in multiobjective traveling salesman and set covering problems. Applied Mathematics and Computation, 237:1 - 19, 2014.

17. R. G. Garroppo, S. Giordano, and L. Tavanti. A survey on multi-constrained optimal path computation: Exact and approximate algorithms. Computer Networks, 54(17):3081 - 3107, 2010. 
18. Y. Y. Haimes, L.S. Lasdon, and D.A. Wismer. On a Bicriterion Formulation of the Problems of Integrated System Identification and System Optimization. IEEE Transactions on Systems, Man, and Cybernetics, 1(3):296-297, July 1971.

19. P. Hansen. Bicriterion path problems. In Günter Fandel and Tomas Gal, editors, Multiple Criteria Decision Making Theory and Application, volume 177 of Lecture Notes in Economics and Mathematical Systems, pages 109-127. Springer Berlin Heidelberg, 1980.

20. John N. Hooker. Integration of $A I$ and $O R$ Techniques in Constraint Programming for Combinatorial Optimization Problems: 10th International Conference, CPAIOR 2013, Yorktown Heights, NY, USA, May 18-22, 2013. Proceedings, chapter Decision Diagrams and Dynamic Programming, pages 94-110. Springer Berlin Heidelberg, Berlin, Heidelberg, 2013.

21. C.-L. Hwang and A.S.M. Masud. Multiple Objective Decision Making Methods and Applications: A state-of-the-art survey, volume 164 of Lecture Notes in Economics and Mathematical Systems. Springer-Verlag Berlin Heidelberg, 1979.

22. IBM ILOG. Cplex optimization studio 12.6.3 user manual, 2016.

23. G. Kirlik and S. Sayın. A new algorithm for generating all nondominated solutions of multiobjective discrete optimization problems. European Journal of Operational Research, 232(3):479 - 488, 2014.

24. M. Laumanns, L. Thiele, and E. Zitzler. An efficient, adaptive parameter variation scheme for metaheuristics based on the epsilon-constraint method. European Journal of Operational Research, 169(3):932 - 942, 2006.

25. C. Y. Lee. Representation of switching circuits by binary-decision programs. Bell Systems Technical Journal, 38:985-999, 1959.

26. Ronald Prescott Loui. Optimal paths in graphs with stochastic or multidimensional weights. Commun. ACM, 26(9):670-676, September 1983.

27. M. Özlen and M. Azizoğlu. Multi-objective integer programming: A general approach for generating all non-dominated solutions. European Journal of Operational Research, 199(1):25 - 35, 2009.

28. K. Miettinen. Nonlinear multiobjective optimization. Kluwer Academic Publishers, Boston, 1999.

29. Melih Özlen, Benjamin A. Burton, and Cameron A. G." MacRae. Multi-objective integer programming: An improved recursive algorithm. Journal of Optimization Theory and Applications, 160(2):470-482, 2013.

30. A. Raith and M. Ehrgott. A comparison of solution strategies for biobjective shortest path problems. Computers \& Operations Research, 36(4):1299 - 1331, 2009.

31. J. Rui Figueira, G. Tavares, and M. M. Wiecek. Labeling algorithms for multiple objective integer knapsack problems. Comput. Oper. Res., 37(4):700-711, April 2010.

32. R. E. Steuer and E.-U. Choo. An interactive weighted tchebycheff procedure for multiple objective programming. Mathematical Programming, 26(3):326-344, 1983.

33. Thomas Stidsen, Kim Allan Andersen, and Bernd Dammann. A branch and bound algorithm for a class of biobjective mixed integer programs. Management Science, 60(4):1009-1032, 2014.

34. E. L. Ulungu and J. Teghem. Multi-objective combinatorial optimization problems: A survey. Journal of Multi-Criteria Decision Analysis, 3(2):83-104, 1994.

35. P. Vincke. Problemes multicriteres. Cahiers Centre Etudes Recherche Operationnelle, 16:425-439, 1974. 
36. I. Wegener. Branching Programs and Binary Decision Diagrams: Theory and Applications. SIAM monographs on discrete mathematics and applications. Society for Industrial and Applied Mathematics, 2000.

37. A. Zhou, B.-Y. Qu, H. Li, S.-Z. Zhao, P. N. Suganthan, and Q. Zhang. Multiobjective evolutionary algorithms: A survey of the state of the art. Swarm and Evolutionary Computation, 1(1):32 - 49, 2011. 